\title{
Impact of Plan-Level Access Restrictions on Effectiveness of Biologics Among Patients with Rheumatoid or Psoriatic Arthritis
}

\author{
Natalie Boytsov ${ }^{1} \cdot$ Xiang Zhang $^{1} \cdot$ Kristin A. Evans ${ }^{2} \cdot$ Barbara H. Johnson ${ }^{2}$
}

Published online: 8 June 2019

(c) The Author(s) 2019

\begin{abstract}
Background Novel disease-modifying antirheumatic drugs (DMARDs) can slow disease progression among patients with rheumatoid arthritis (RA) and psoriatic arthritis (PsA); however, some health plans require prior authorization (PA) or step therapy for access to treatments.

Objectives This retrospective study compared treatment effectiveness among RA and PsA patients with and without planlevel access restrictions to biologic DMARDs (bDMARDs) or targeted synthetic DMARDs (tsDMARDs). Medication adherence, a component of effectiveness, was also examined as a secondary outcome.

Methods RA and PsA patients aged 18-64 years with one or more claims for subcutaneous bDMARDs between January 1, 2014 and December 31, 2015, with plan-level access data available, were identified within the IBM MarketScan claims database. The primary outcome was treatment effectiveness assessed during the 12 months following the first qualifying DMARD claim. Multivariate modeling examined the correlation between access restrictions and treatment effectiveness. Medication adherence during the 12-month follow-up period was also compared between patients with and without access restrictions. Results Among 3993 RA and 1713 PsA patients, 34.2 and 35.1\%, respectively, had access restrictions, of whom 70.5 and $78.9 \%$, respectively, had plans with step therapy. Compared with patients whose plans did not require step therapy, odds of treatment effectiveness were 19\% lower (odds ratio [OR] 0.81, 95\% CI: 0.67-0.98; $p=0.033$ ) for RA patients and 27\% lower (OR 0.73, 95\% CI: 0.55-0.98; $p=0.037$ ) for PsA patients in plans with step therapy. Differences in effectiveness were driven by differences in medication adherence, the odds of which were $19 \%$ lower (OR 0.81, 95\% CI 0.68-0.96; $p=0.014$ ) among RA patients and $29 \%$ lower (OR 0.71, 95\% CI: 0.54-0.94; $p=0.017$ ) among PsA patients in plans with versus without step therapy.

Conclusions Compared with patients in plans without access restrictions or with PA only, RA and PsA patients in insurance plans with step therapy had lower odds of treatment effectiveness, mainly due to lower odds of adhering to treatment, during the 12 months following subcutaneous bDMARD initiation.
\end{abstract}

\section{Introduction}

Rheumatoid arthritis (RA) is one of the most common autoimmune conditions in the US, affecting approximately 1.3 million American adults, with approximately 41 new cases

Electronic supplementary material The online version of this article (https://doi.org/10.1007/s41669-019-0152-1) contains supplementary material, which is available to authorized users.

Natalie Boytsov

boytsov_natalie_n@lilly.com

1 Eli Lilly and Company, Lilly Corporate Center, Indianapolis, IN 46285, USA

2 IBM Watson Health, 75 Binney St, Cambridge, MA 02142, USA

\section{Key Points for Decision Makers}

Patient access to biologic DMARDs (bDMARDs) and targeted synthetic DMARDs (tsDMARDs) may impact medication treatment effectiveness by lowering the odds of medication adherence.

A step-therapy approach to bDMARDs or tsDMARDs has an adverse impact on treatment effectiveness that might lead to higher healthcare resource use.

Payers might consider improving patient access to various bDMARDs and tsDMARDs to improve patient adherence and overall treatment effectiveness. 
per 100,000 people each year [1-3]. Symptoms include painful swelling of the joints, typically starting in the hands and feet and leading to bone erosion, physical limitations and disability $[4,5]$. The societal and economic burden of RA is substantial, with a work disability rate of more than one-third within 10 years, and over $\$ 8$ billion in RA-related healthcare costs annually $[5,6]$. Psoriatic arthritis (PsA) occurs in up to $24 \%$ of the approximately 7 million American adults living with psoriasis [7, 8], and, for many, shares some of the clinical features of RA, although is generally milder in presentation [9]. Indirect cost estimates of $\$ 11,000$ and direct cost estimates of between $\$ 12,000$ and $\$ 24,000$ per patient are attributable to PsA annually $[10,11]$.

Both conditions are immune-mediated, and patient outcomes have benefited from the rapid advances in novel disease-modifying antirheumatic drugs (DMARDs), which reduce inflammation and slow disease progression [12]. The conventional synthetic DMARD (csDMARD) methotrexate, is the preferred first-line therapy due to its history of clinical success, ease of use, and low cost [13]; however, most patients will discontinue therapy within $3-5$ years due to disease progression or adverse events $[14,15]$. For patients with RA, when methotrexate is deemed insufficient for disease control, the American College of Rheumatology (ACR) recommends adding either an additional csDMARD or initiating a biologic DMARD (bDMARD) with or without continuation of methotrexate [16]. If low disease activity or remission is not initially achieved, the guidelines expand to include targeted synthetic DMARDs (tsDMARD) or any combination of the previously mentioned drug categories.

However, it may be difficult for some patients and their physicians to optimize therapy for RA or PsA as many insurance formularies dictate treatment choices by requirement of a prior authorization (PA) before a drug could be covered, or a stepped approach to drug therapy in which multiple rounds of less expensive drugs must be prescribed before other, more costly therapies, such as a bDMARD, could be covered. RA and PsA are heterogeneous diseases, therefore one patient may not respond to the same therapy as another patient. Treatment guidelines advocate for a treat-to-target strategy, which should allow for selection of an optimal treatment choice [12]. The impact of such formulary restrictions on treatment outcomes among RA and PsA patients is unclear. The main objective of this study was to compare treatment effectiveness between patients prescribed a subcutaneous biologic with and without health plan-level bDMARD or tsDMARD access restrictions. Medication adherence, a component of treatment effectiveness, was also compared between groups.

\section{Methods}

\subsection{Data Source}

Data for this study were drawn from the IBM MarketScan ${ }^{\circledR}$ Commercial Claims and Encounters Database (IBM Corporation, Armonk, NY, USA), which contains the inpatient, outpatient medical, and outpatient prescription drug claims of approximately 137.6 million employees and their dependents, covered under a variety of fee-for-service and managed care health plans between 1995 and 2017, including 24.4 million lives in 2016 (the follow-up year of the current study). The pharmacy benefit plan formulary data were provided by Managed Markets Insights and Technology, Inc. (MMIT, Yardley, PA, USA). MMIT's database contains information on formulary design for the treatments of interest, by plan provider and type. Patient data from MarketScan were linked with formulary data from the MMIT database by plan provider and type to determine patient-level drug access restrictions.

\subsection{Study Population}

Patients meeting the following inclusion criteria were eligible for study inclusion (Fig. 1): (1) at least one claim for a bDMARD or tsDMARD between January 1, 2014 and December 31, 2015 (study period), and the date of the first prescription during the study period was the patient's index date; (2) at least 18 years of age on the index date; (3) at least 6 months of continuous enrollment in an insurance plan with medical and pharmacy benefits prior to the index date (baseline period); (4) at least one non-diagnostic medical claim with a diagnosis code for RA or PsA during the baseline period (Online Resource 1); and (5) having continuous benefit enrollment in the same insurance carrier from the index date through the 12-month follow-up period. Patients were excluded from the study if they had any claims for the index drug during the baseline period, or any non-diagnostic claims with a diagnosis of ankylosing spondylitis, Crohn's disease, juvenile idiopathic arthritis, or ulcerative colitis any time during the study period. RA patients with any claims for apremilast, secukinumab, or ustekinumab during the study period were excluded, as were PsA patients with any claims with a diagnosis code for RA during the baseline period.

Patients meeting all the above criteria were stratified into two mutually exclusive cohorts, i.e. RA with or without PsA, and PsA only. Patients were linked to health plan-level formulary structure data to determine whether their insurance plans had access restrictions to bDMARDs or tsDMARDs. Since adherence is part of the definition of treatment effectiveness used in this study [19], only patients who indexed 


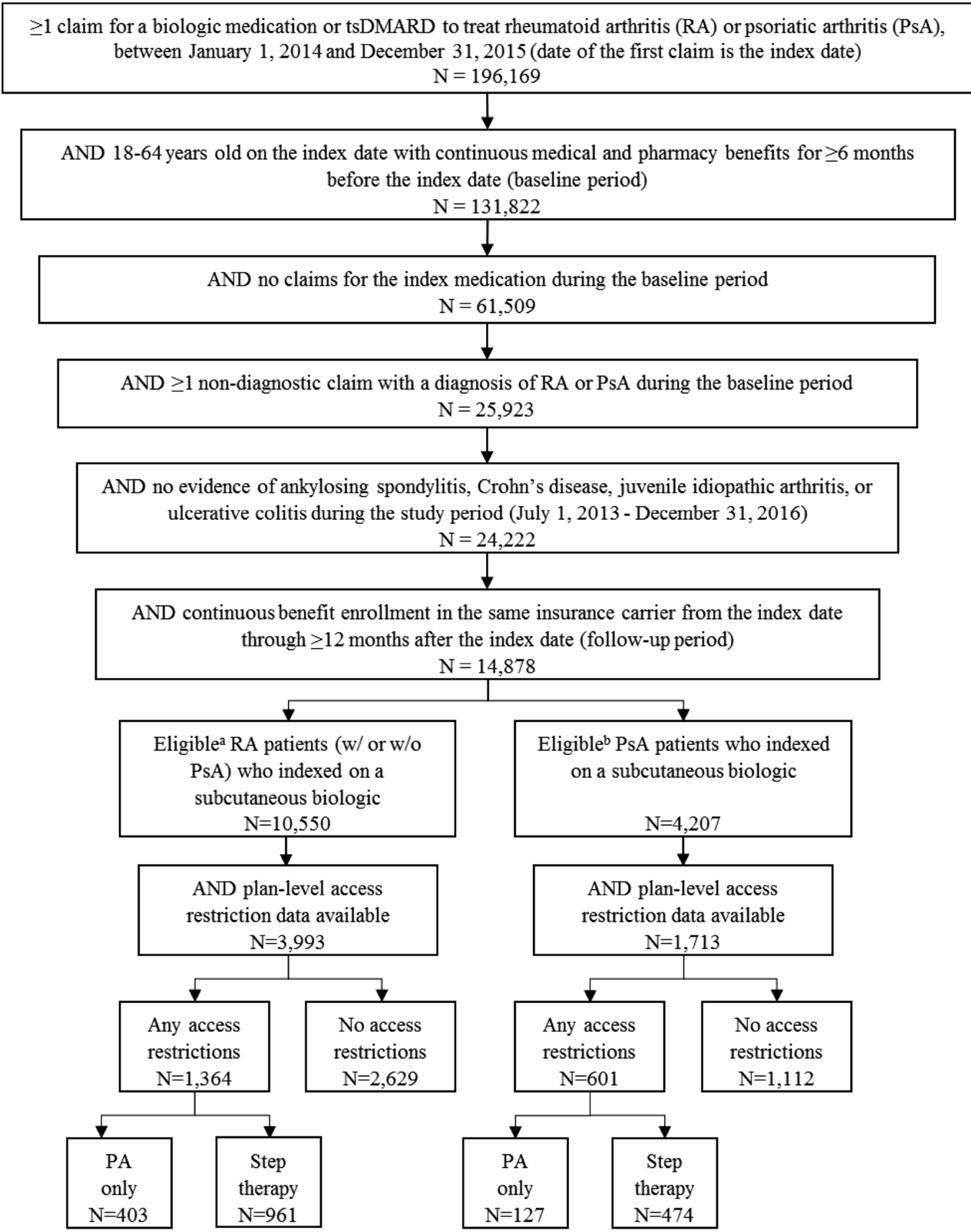

Fig. 1 Patient selection. a No prescription for PsA-only treatment (apremilast, secukinumab, or ustekinumab) during the study period. b No prescription for RA-only treatment (anakinra, rituximab or tocilizumab) during the study period. $b D M A R D$ biologic disease-

on a subcutaneous medication were included in the final analysis as route of administration has been shown to impact treatment adherence $[17,18]$. However, access restriction classification was based on restrictions to either bDMARDs modifying antirheumatic drug, tsDMARD targeted synthetic diseasemodifying antirheumatic drug, $P S A$ psoriatic arthritis, $R A$ rheumatoid arthritis

or tsDMARDs since restricted access to either indicates that patients cannot freely switch to other types of drugs. Ultimately, patients who indexed on one of the following subcutaneous bDMARDs were included: abatacept, adalimumab, 
anakinra (RA only), certolizumab, etanercept, golimumab, secukinumab (PsA only), tocilizumab (RA only), or ustekinumab (PsA only). If a patient's plan imposed either PA or step therapy on any bDMARD or tsDMARD medication indicated for RA or PsA, that patient was considered to have access restrictions. Patient cohorts were then further stratified by the presence of any access restrictions, as well as by the type of access restriction, resulting in the following cohorts: any access restrictions, PA only, step therapy with or without PA, and no access restrictions.

\subsection{Variables}

The access restrictions described above (any access restrictions, PA only, step therapy with or without PA vs. no access restrictions) were the primary explanatory variable in this study. The main outcome of interest was treatment effectiveness during the follow-up period. A claims-based algorithm, defined and validated by Curtis et al. using the Disease Activity Score 28 (DAS28) as the gold standard, and used in prior research regarding biologic effectiveness, was used in this study to assess treatment effectiveness [19-24]. Validation of this algorithm required that algorithm-defined effectiveness be associated with high adherence to the medication and a DAS28 score indicating low disease activity, or an improvement of at least 1.2 units in the DAS28 score. The requirement for high adherence assured that qualifying DAS28 scores could confidently be attributed to the medication [19]. Patients were considered to have been treated effectively with their index medication if they met all of the following six criteria during the 12-month follow-up period: (1) were highly adherent to the index medication, defined as having a proportion of days covered (PDC) of $\geq 80 \%$ for the index medication during the 12-month follow-up period (PDC calculated as the sum of days of supply for all prescription claims divided by 365 days); (2) did not switch to a new bDMARD or tsDMARD; (3) did not have a new csDMARD (one not prescribed during the 6-month baseline period) added to their medication regimen; (4) did not have an increase in the dose or frequency of their index drug; (5) had fewer than two intra-articular glucocorticoid injections after the third month of follow-up; and (6) had no more than 30 days of an oral glucocorticoid after the third month of follow-up (for those with no oral glucocorticoid prescription in the baseline period), or had a cumulative dose increase of $120 \%$ or less between the 6-month baseline period and the last 6 months of the follow-up period (for those with an oral glucocorticoid prescription in the baseline period). Adherence to the index medication was also examined as a secondary outcome, and the frequency of each of the other treatment patterns described above are also reported.
Patient demographic and clinical characteristics were assessed on the index date and during the baseline period, respectively. Demographic characteristics including age, sex, geographic region, urban residence, and insurance plan type were measured on the index date. Clinical characteristics including the Deyo-Charlson Comorbidity Index (DCI; higher values indicated a higher number of select comorbidities) [25], select comorbid conditions (anemia, anxiety, cardiovascular disease, chronic respiratory condition, depression, diabetes [type 1 or 2], dyslipidemia, fibromyalgia, fragility fracture, hypertension, low back pain, malignancy, osteoarthritis, osteoporosis, psoriasis), and select medications (bDMARDs, csDMARDs, tsDMARDs, glucocorticoids, antidepressants, antidiabetics, antihyperlipidemics, antihypertensives, or pain medications, i.e. nonsteroidal antiinflammatory drugs [NSAIDs], opioids, other) were measured during the baseline period. Additionally, the number of prescription claims for glucocorticoids, antidepressants, and pain medications were annualized and reported per person per year (PPPY).

Select healthcare resource utilization and costs were also assessed during the baseline and follow-up periods between access restriction cohorts. The proportion of patients with an inpatient admission, emergency room (ER) visit, and RArelated office visit in the baseline period were estimated. Baseline medical costs (associated with inpatient and outpatient medical claims), outpatient prescription costs, and total healthcare costs (medical plus outpatient prescription costs) were annualized and reported PPPY. Costs are the paid amounts of fully adjudicated claims, including insurer and health plan payments, as well as patient cost-sharing in the form of co-payment, deductible, and co-insurance. All costs were inflated to 2016 US dollars using the medical care component of the Consumer Price Index.

\subsection{Statistical Analysis}

Mean, standard deviation (SD) and median were reported for continuous variables, while frequencies and percentages were reported for categorical variables. Bivariate comparisons, using Chi square tests for categorical variables and $t$-tests for continuous variables, were made between the 'any access restrictions' and 'no access restrictions' cohorts. RA and PsA patients were analyzed separately.

Multivariate analysis examined differences in the main outcome of treatment effectiveness between patients with and without access restrictions, while controlling for baseline demographic, clinical and economic characteristics. Two mutually exclusive cohorts (RA and PsA) were analyzed separately in all statistical models. Separate models were constructed with any access restrictions, PA only, and step therapy (with or without PA) as the main explanatory variables. Medication adherence was also examined as a 
secondary outcome in multivariate models. Sixteen models were constructed, and each model was adjusted for age, sex, geographic region, urban residence, health plan type, index year, select comorbid conditions (excluding fragility fractures due to low prevalence), baseline bDMARD or tsDMARD use, baseline glucocorticoid use, total baseline healthcare costs, and the number of rheumatologist visits in the baseline period. Odds ratios (ORs), 95\% confidence intervals $(\mathrm{CI})$, and $p$-values were produced by logistic regression models, indicating the relative likelihood of medication adherence or treatment effectiveness associated with having each level of access restrictions, compared with all other levels of restrictions (i.e. any restrictions vs. no restrictions; PA only vs. step therapy or no restrictions; step therapy vs. PA only or no restrictions). All analyses were performed using $\mathrm{R}$ statistical software version 3.5.0 (The R Foundation for Statistical Computing, Vienna, Austria; ISBN 3-900051-07-0, URL http://www.R-project.org/). A $p$ value $<0.05$ was considered statistically significant. $P$-values were not adjusted for multiple comparisons.

\section{Results}

A total of 3993 RA patients and 1713 PsA patients in the MarketScan databases met all eligibility criteria and were included in the analysis (Fig. 1). These patients were distributed across a total of 101 insurance plans, $85 \%$ of which were national (vs. regional) plans. Approximately one-third of eligible patients (1364 [34.2\%] RA patients and 601 [35.1\%] PsA patients) from 25 (23\%) different insurance plans, had access restrictions to at least one bDMARD or tsDMARD to treat RA or PsA. Among patients with access restrictions, 961 (70.5\%) RA patients and 474 (78.9\%) PsA patients were in plans that required step therapy, with or without PA, and 403 (29.5\%) RA patients and 127 (21.1\%) PsA patients were in plans that required PA only. The remaining 2629 (65.8\%) RA patients and 1112 (64.9\%) PsA patients with no access restrictions to any of the study medications represented $82(77 \%)$ different insurance plans.

\subsection{Baseline Characteristics of Rheumatoid Arthritis (RA) Patients}

Baseline demographic and clinical characteristics of RA patients are shown in Table 1 and in Online Resource 2. RA patients were predominately female $(77.7 \%)$ and, on average, $50.1 \pm 9.8$ years of age on their index date (Table 1$)$. The majority resided in the southern region of the US (53.2\%), lived in an urban area (85.7\%), and were enrolled in an exclusive provider organization (EPO) or preferred provider organization (PPO) insurance plan (56.9\%) during the study period (Online Resource 2).
The average DCI was $1.4 \pm 0.8$, and the most common comorbid conditions of interest were hypertension (27.2\%), osteoarthritis (23.1\%), and dyslipidemia (14.3\%). Patients with any access restrictions had a higher DCI compared with patients with no restrictions $(1.4 \pm 0.9$ vs. $1.3 \pm 0.8$; $p<0.001)$. Some comorbid conditions were also significantly more common among patients with any access restrictions, compared with no access restrictions, including chronic respiratory conditions, diabetes, dyslipidemia, hypertension, and osteoarthritis (all $p<0.05$ ).

Select baseline medication and healthcare utilization are reported in Table 2. RA patients with access restrictions were more likely to have at least one csDMARD or glucocorticoid prescription claim during the baseline period, and had significantly more glucocorticoid prescription claims, compared with those without access restrictions (all $p<0.05$ ). Patients with any access restrictions were also more likely to have at least one prescription for an antidiabetic medication or an antihypertensive medication during the baseline period compared with patients with no access restrictions (both $p<0.01$ ). Similar proportions of patients with and without access restrictions had at least one prescription for an antidepressant, although patients with access restrictions had significantly more antidepressant prescription claims per patient than those without access restrictions $(p<0.001)$. There was no significant difference in the proportion of patients with at least one prescription claim for opioids, NSAIDs, or other pain medications; however, patients with access restrictions filled significantly more NSAID prescriptions per patient than those without access restrictions $(p<0.001)$. Similar proportions of patients with and without access restrictions had at least one inpatient admission (any cause and infection-related) and at least one RA-related office visit in the baseline period. A significantly higher proportion of patients with access restrictions had at least one ER visit in the baseline period compared with those without access restrictions $(8.9 \%$ vs. $4.8 \% ; p<0.001)$, and there were no significant differences in medical costs, outpatient prescription costs, or total healthcare costs in the baseline period between patients with and without access restrictions.

\subsection{Treatment Patterns of RA Patients}

The unadjusted results for treatment effectiveness, as well as medication adherence and the frequency of specific treatment modifications, are shown in Table 3. In the unadjusted analysis, patients with any access restrictions were less likely to meet the definition of treatment effectiveness with their index medication during the 12-month follow-up period compared with patients with no access restrictions $(20.0 \%$ vs. $22.8 \% ; p=0.045)$. The mean PDC for RA patients 
Table 1 Baseline demographic and clinical characteristics of rheumatoid arthritis patients

\begin{tabular}{|c|c|c|c|c|}
\hline & \multirow{2}{*}{$\begin{array}{l}\text { All RA } \\
\text { patients } \\
{[N=3993]}\end{array}$} & \multicolumn{2}{|c|}{ Access restrictions } & \multirow[t]{2}{*}{$p$ value (any vs. none) } \\
\hline & & Any $[n=1364]$ & None $[n=2629]$ & \\
\hline Age, years (mean [SD]) & $50.1[9.8]$ & $50.4[9.6]$ & $50.0[9.8]$ & 0.294 \\
\hline \multicolumn{5}{|l|}{ Age group, years } \\
\hline $18-34$ & $360(9.0)$ & $115(8.4)$ & $245(9.3)$ & 0.353 \\
\hline $35-44$ & $649(16.3)$ & $209(15.3)$ & $440(16.7)$ & 0.251 \\
\hline $45-54$ & $1356(34.0)$ & $479(35.1)$ & $877(33.4)$ & 0.266 \\
\hline $55-64$ & $1628(40.8)$ & $561(41.1)$ & 1067 (40.6) & 0.740 \\
\hline Male & $889(22.3)$ & $313(22.9)$ & $576(21.9)$ & 0.455 \\
\hline \multicolumn{5}{|l|}{ Region } \\
\hline Northeast & $484(12.1)$ & $132(9.7)$ & $352(13.4)$ & $0.001^{*}$ \\
\hline North Central & $845(21.2)$ & $192(14.1)$ & $653(24.8)$ & $<0.001 * *$ \\
\hline South/unknown & $2131(53.4)$ & $845(62.0)$ & $1286(48.9)$ & $<0.001 * *$ \\
\hline West & $533(13.3)$ & $195(14.3)$ & 338 (12.9) & 0.205 \\
\hline Urban residence & $3422(85.7$ & $1050(77.0)$ & $2372(90.2)$ & $<0.001 * *$ \\
\hline $\mathrm{EPO} / \mathrm{PPO}$ insurance & $2272(56.9)$ & 749 (54.9) & $1523(57.9)$ & 0.068 \\
\hline DCI (mean [SD]) & $1.4[0.8]$ & $1.4[0.9]$ & $1.3[0.8]$ & $<0.001 * *$ \\
\hline \multicolumn{5}{|l|}{ Comorbid conditions } \\
\hline Anemia & $266(6.7)$ & $96(7.0)$ & $170(6.5)$ & 0.492 \\
\hline Anxiety & $292(7.3)$ & $108(7.9)$ & $184(7.0)$ & 0.290 \\
\hline Cardiovascular disease & $233(5.8)$ & $85(6.2)$ & $148(5.6)$ & 0.441 \\
\hline Chronic respiratory condition & $406(10.2)$ & $160(11.7)$ & $246(9.4)$ & $0.019^{*}$ \\
\hline Depression & $376(9.4)$ & $137(10.0)$ & $239(9.1)$ & 0.328 \\
\hline Diabetes (type 1 or 2 ) & $496(12.4)$ & $206(15.1)$ & $290(11.0)$ & $<0.001 * *$ \\
\hline Dyslipidemia & $571(14.3)$ & $221(16.2)$ & $350(13.3)$ & $0.013 *$ \\
\hline Fibromyalgia & 464 (11.6) & $154(11.3)$ & $310(11.8)$ & 0.639 \\
\hline Fragility fracture & $15(0.4)$ & $7(0.5)$ & $8(0.3)$ & 0.306 \\
\hline Hypertension & $1087(27.2)$ & $444(32.6)$ & $643(24.5)$ & $<0.001 * *$ \\
\hline Low back pain & $408(10.2)$ & $147(10.8)$ & $261(9.9)$ & 0.401 \\
\hline Malignancy & $111(2.8)$ & $36(2.6)$ & $75(2.9)$ & 0.697 \\
\hline Osteoarthritis & $924(23.1)$ & $362(26.5)$ & $562(21.4)$ & $<0.001 * *$ \\
\hline Osteoporosis & $172(4.3)$ & $62(4.5)$ & $110(4.2)$ & 0.594 \\
\hline Psoriasis & $104(2.6)$ & $39(2.9)$ & $65(2.5)$ & 0.467 \\
\hline
\end{tabular}

Data are expressed as $n(\%)$ unless otherwise specified

$S D$ standard deviation, EPO/PPO exclusive provider organization/preferred provider organization, $D C I$ Deyo-Charlson Comorbidity Index, $R A$ rheumatoid arthritis, ${ }^{*} p<0.05$, ** $p<0.001$ with any access restrictions was significantly lower than among those without access restrictions (51.8\% vs. $55.7 \%$; $p<0.001)$. Patients with any access restrictions to RA medications were less likely to have high adherence to their index medication than those without access restrictions $(25.8 \%$ vs. $29.3 \% ; p<0.001)$. There was no statistically significant difference between patients with and without access restrictions for any of the other criteria of the treatment effectiveness algorithm. The most common treatment modification among RA patients was switching to a new bDMARD or tsDMARD (16.6\%).

After controlling for patient demographic and clinical characteristics, having any access restrictions (PA or step therapy) was not significantly associated with the odds of treatment effectiveness (OR 0.93, 95\% CI 0.78-1.10; $p=0.376$ ) among RA patients receiving subcutaneous bDMARDs (Fig. 2). However, among the subset of patients whose access restrictions included step therapy (with or without PA), the odds of treatment effectiveness during 12 months of follow-up were $19 \%$ lower (OR $0.81,95 \%$ CI $0.67-0.98 ; p=0.033)$ compared with all other groups, and $17 \%$ lower (OR 0.83 , 95\% CI 0.68-1.01; $p=0.06$ ) when compared with patients with no access restriction. Similarly, having any access restrictions was not significantly associated with the odds of medication adherence (OR 0.90 , 95\% CI $0.77-1.05 ; p=0.182$ ), but the odds of adherence 
Table 2 Baseline outpatient prescription drug use, healthcare resource utilization and costs for rheumatoid arthritis patients

All RA patients $[N=3993]$ Access restrictions $p$ value (any vs. none) Any $[n=1364] \quad$ None $[n=2629]$

\begin{tabular}{|c|c|c|c|c|}
\hline \multicolumn{5}{|l|}{ Medications } \\
\hline bDMARD & $157(3.9)$ & $63(4.6)$ & $94(3.6)$ & 0.108 \\
\hline tsDMARD & $11(0.3)$ & $4(0.3)$ & $7(0.3)$ & 0.877 \\
\hline csDMARD & $3046(76.3)$ & $1073(78.7)$ & $1973(75.0)$ & $0.011^{*}$ \\
\hline Glucocorticoid & $2802(70.2)$ & $998(73.2)$ & $1804(68.6)$ & $0.003 *$ \\
\hline $\begin{array}{l}\text { Mean [SD] number of glucocorticoid claims } \\
\text { PPPY }\end{array}$ & $6.6[4.8]$ & $7.0[5.0]$ & $6.4[4.6]$ & $0.001 *$ \\
\hline Antidepressant & $1259(31.5)$ & $453(33.2)$ & $806(30.7)$ & 0.100 \\
\hline $\begin{array}{l}\text { Mean }[S D] \text { number of antidepressant claims } \\
\text { PPPY }\end{array}$ & $7.5[5.5]$ & $8.9[6.2]$ & $6.8[4.9]$ & $<0.001 * *$ \\
\hline Antidiabetic & $391(9.8)$ & $157(11.5)$ & $234(8.9)$ & $0.009^{*}$ \\
\hline Antihyperlipidemic & 796 (19.9) & $287(21.0)$ & 509 (19.4) & 0.208 \\
\hline Antihypertensive & $1465(36.7)$ & $543(39.8)$ & $922(35.1)$ & $0.003^{*}$ \\
\hline Pain medication & $2595(65.0)$ & $900(66.0)$ & $1695(64.5)$ & 0.343 \\
\hline Opioid & $1764(44.2)$ & $611(44.8)$ & $1153(43.9)$ & 0.571 \\
\hline Mean $[\mathrm{SD}]$ number of opioid claims PPPY & $7.9[7.5]$ & $8.1[7.8]$ & $7.7[7.3]$ & 0.370 \\
\hline NSAID & $1761(44.1)$ & $618(45.3)$ & $1143(43.5)$ & 0.269 \\
\hline Mean [SD] number of NSAID claims PPPY & $5.4[3.7]$ & $5.8[4.2]$ & $5.1[3.5]$ & $<0.001 * *$ \\
\hline Other pain medication & 705 (17.7) & $253(18.5)$ & $452(17.2)$ & 0.287 \\
\hline $\begin{array}{l}\text { Mean }[\mathrm{SD}] \text { number of other pain medication } \\
\text { claims PPPY }\end{array}$ & $4.9[4.0]$ & $5.1[3.6]$ & $4.9[4.2]$ & 0.550 \\
\hline \multicolumn{5}{|l|}{ Healthcare utilization } \\
\hline IP admission & $202(5.1)$ & $61(4.5)$ & $141(5.4)$ & 0.223 \\
\hline IP admission due to infection & $27(0.7)$ & $8(0.6)$ & $19(0.7)$ & 0.618 \\
\hline Emergency room visits & $248(6.2)$ & $122(8.9)$ & $126(4.8)$ & $<0.001 * *$ \\
\hline RA-related office visits & $2329(58.3)$ & $791(58.0)$ & $1538(58.5)$ & 0.757 \\
\hline \multicolumn{5}{|l|}{ Healthcare costs PPPY } \\
\hline Medical costs (mean [SD]) & $\$ 5900[\$ 25,273]$ & $\$ 5362[\$ 24,285]$ & $\$ 6179[\$ 25,771]$ & 0.333 \\
\hline Medical costs (median) & $\$ 1321$ & $\$ 1255$ & $\$ 1355$ & \\
\hline OP prescription costs (mean $[\mathrm{SD}]$ ) & $\$ 4563[\$ 14,045]$ & $\$ 4247[\$ 9501]$ & $\$ 4727[\$ 15,899]$ & 0.306 \\
\hline OP prescription costs (median) & $\$ 1656$ & $\$ 1798$ & $\$ 1569$ & \\
\hline Total healthcare costs (mean [SD]) & $\$ 10,463[\$ 29,738]$ & $\$ 9609[\$ 26,361]$ & $\$ 10,906[\$ 31,343]$ & 0.191 \\
\hline Total healthcare costs (median) & $\$ 3756$ & $\$ 3716$ & $\$ 3801$ & \\
\hline
\end{tabular}

Data are expressed as $n(\%)$ unless otherwise specified

$D M A R D$ disease-modifying antirheumatic drug, $b D M A R D$ biologic DMARD, tsDMARD targeted synthetic DMARD, $c s D M A R D$ conventional synthetic DMARD, $S D$ standard deviation, $P P P Y$ per patient per year, NSAID nonsteroidal anti-inflammatory drug, $R A$ rheumatoid arthritis, $I P$ inpatient, $O P$ outpatient, $* p<0.05, * * p<0.001$

were $19 \%$ lower (OR 0.81, 95\% CI 0.68-0.96; $p=0.014$ ) for patients with step therapy requirements compared with all other restriction groups, and $18 \%$ lower (OR $0.82,95 \% \mathrm{CI}$ 0.69-0.98; $p=0.025$ ) when compared with patients with no restrictions. Baseline factors that were significantly associated with lower odds of treatment effectiveness and medication adherence included younger age, female sex, living in the south, and a baseline diagnosis of anxiety or depression (Fig. 3 and Online Resource 3). Patients with diabetes, fibromyalgia, hypertension, or baseline glucocorticoid use had lower odds of treatment effectiveness, while those who filled prescriptions for a bDMARD or tsDMARD in the baseline period had higher odds of medication adherence.

\subsection{Healthcare Resource Utilization and Costs for RA Patients}

The unadjusted results for healthcare resource utilization and costs measured in the 12-month follow-up period are shown in Table 4 . While patients with any 
Table 3 Treatment patterns of rheumatoid arthritis patients from the index date through the 12-month follow-up period

\begin{tabular}{|c|c|c|c|c|}
\hline & \multirow{2}{*}{$\begin{array}{l}\text { All RA patients } \\
{[N=3993]}\end{array}$} & \multicolumn{2}{|c|}{ Access restrictions } & \multirow[t]{2}{*}{$p$ value (any vs. none) } \\
\hline & & Any $[n=1364]$ & None $[n=2629]$ & \\
\hline Effective treatment ${ }^{\mathrm{a}}$ & $872(21.8)$ & $273(20.0)$ & $599(22.8)$ & $0.045^{*}$ \\
\hline $\mathrm{PDC}, \%$ (mean $[\mathrm{SD}])$ & $54.3[30.8]$ & $51.8[30.9]$ & $55.7[30.7]$ & $<0.001 * *$ \\
\hline Adherent (PDC $\geq 80 \%)$ & $1122(28.1)$ & $352(25.8)$ & $770(29.3)$ & $0.020^{*}$ \\
\hline \multicolumn{5}{|l|}{ Treatment modifications } \\
\hline One or more treatment modifications & $1143(28.6)$ & $389(28.5)$ & $754(28.7)$ & 0.915 \\
\hline Days to first treatment modification (mean [SD]) & $145.2[98.4]$ & $145.1[99.8]$ & $145.2[97.7]$ & 0.991 \\
\hline Addition of a new csDMARD & $316(7.9)$ & $103(7.6)$ & $213(8.1)$ & 0.541 \\
\hline Increase in the index drug dose $\mathrm{b}^{\mathrm{b}}$ & $92(2.3)$ & $40(2.9)$ & $52(2.0)$ & 0.057 \\
\hline Switch to a new bDMARD or tsDMARD & $662(16.6)$ & $230(16.9)$ & $432(16.4)$ & 0.729 \\
\hline Number of switches (mean [SD]) & $1[1.4]$ & $2[1.6]$ & $1[1.3]$ & 0.306 \\
\hline Addition of a new glucocorticoid, or increase in dose & $164(4.1)$ & $63(4.6)$ & $101(3.8)$ & 0.241 \\
\hline Two or more intra-articular corticosteroid injections ${ }^{c}$ & $242(6.1)$ & $88(6.5)$ & $154(5.9)$ & 0.456 \\
\hline Discontinuation $^{\mathrm{d}}$ of index drug & $3026(75.8)$ & $1052(77.1)$ & $1974(75.1)$ & 0.153 \\
\hline Restart index drug after $\geq 30$-day gap & $824(20.6)$ & $261(19.1)$ & $563(21.4)$ & 0.091 \\
\hline
\end{tabular}

Data are expressed as $n(\%)$ unless otherwise specified

$S D$ standard deviation, DMARD disease-modifying antirheumatic drug, csDMARD conventional synthetic DMARD, $B D M A R D$ biologic DMARD, tsDMARD targeted synthetic DMARD, $R A$ rheumatoid arthritis, $P D C$ proportion of days covered, ${ }^{*} p<0.05, * * p<0.001$

${ }^{a} \mathrm{PDC} \geq 80 \%$ and the absence of any of the listed treatment modifications

${ }^{b}$ Defined separately for each medication using a validated algorithm [18]

${ }^{\mathrm{c}}$ Corticosteroid injections during months $4-12$ of follow-up

${ }^{\mathrm{d}} \geq 30$-day gap in days of supply

\section{Medication adherence}

Any access restrictions vs. no access restrictions PA only vs. step therapy or no access restrictions Step therapy vs. no step therapy

Step therapy vs. no access restrictions

\section{Treatment effectiveness}

Any access restrictions vs. no access restrictions PA only vs. step therapy or no access restrictions Step therapy vs. no step therapy Step therapy vs. no access restrictions
OR $(95 \% \mathrm{Cl}) \quad P$ value

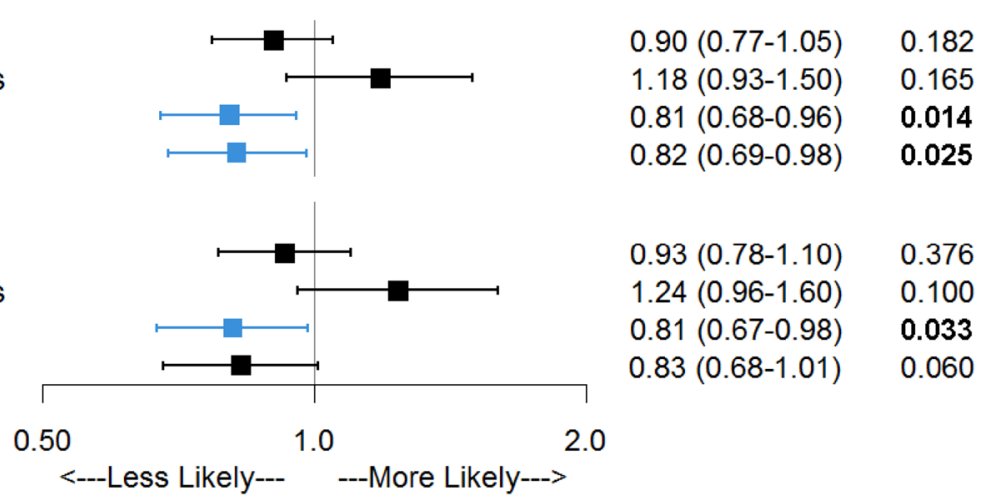

Note: blue shading indicates statistical significance.

Fig. 2 Odds of treatment effectiveness and medication adherence for rheumatoid arthritis patients with different types of access restrictions. Blue shading and bolded $p$ values indicate statistical significance $(p<0.05)$. $C I$ confidence interval, $O R$ odds ratio, $P A$ prior authorization

access restriction were only slightly more likely to have any inpatient admission during the 12-month follow-up period compared with patients without access restrictions (7.8\% vs. $7.0 \% ; p=0.330)$, they were three times as likely to have an inpatient admission for infection than those without access restrictions $(2.3 \%$ vs. $0.8 \% ; p<0.001)$. Consistent with baseline, almost twice as many patients with any access restrictions had at least one visit to the ER during follow-up compared with those without access restrictions $(12.2 \%$ vs. $6.8 \% ; p<0.001)$. Patients without access restrictions had $7.4 \%$ higher total healthcare costs than those with access restrictions $(p=0.003)$, which were mainly driven by significant differences in pharmacy costs, overall and per prescription. 


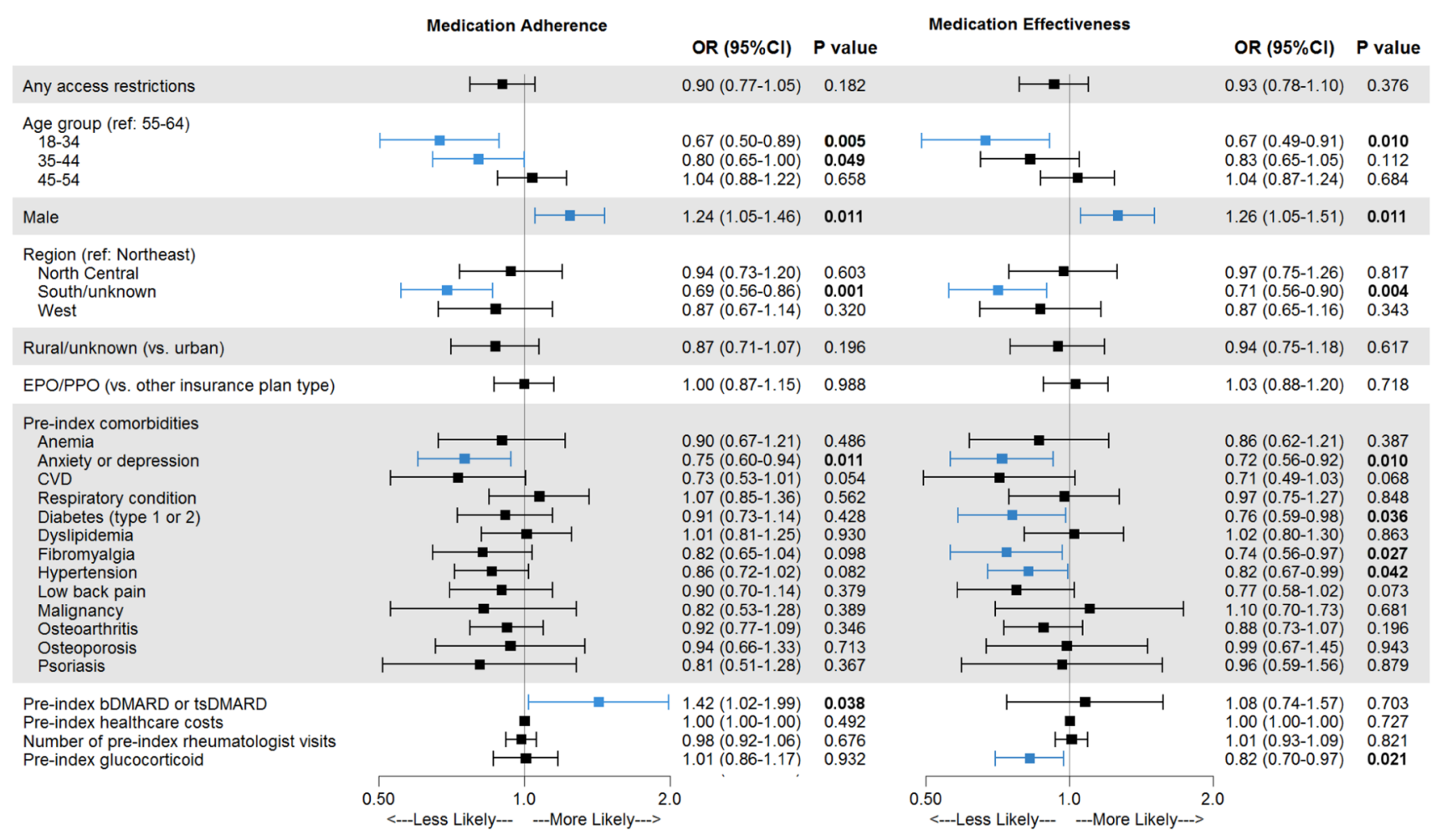

Note: blue shading indicates statistical significance.

Fig. 3 Multivariate analysis of factors influencing treatment effectiveness and medication adherence for rheumatoid arthritis patients. Blue shading and bolded $p$ values indicate statistical significance $(p<0.05)$. bDMARD biologic DMARD, $C I$ confidence interval,

\subsection{Psoriatic Arthritis Patients}

The analysis of 1713 patients diagnosed with PsA found similar results as those for RA patients. Baseline characteristics, including demographics, comorbidities, concomitant medications, healthcare resource utilization, and costs, are reported in Online Resource 4. In brief, PsA patients were slightly younger than their RA counterparts $(47.4 \pm 10.4$ years of age), less likely to be female $(53.9 \%)$, and had a lower DCI $(0.3 \pm 0.8)$. Approximately half (54.3\%) of the PsA cohort had a claim for psoriasis during the 6-month baseline period, and the prevalence was slightly higher among patients with any access restrictions compared with those without access restrictions $(57.7 \%$ vs. $52.4 \% ; p=0.035)$. In addition, hypertension and depression were more common among patients with access restrictions (both $p<0.05$ ).

Among PsA patients, those with any access restrictions were more likely to have at least one outpatient prescription claim for a csDMARD, glucocorticoid, or NSAID (all $p<0.05$ ) than those with no access restrictions in the baseline period (Online Resource 3 ). Patients with access
$D M A R D$ disease-modifying antirheumatic drug, EPO exclusive provider organization, $O R$ odds ratio, $P P O$ preferred provider organization, tsDMARD targeted synthetic DMARD

restrictions also had more frequent inpatient admissions in the baseline period than those without access restrictions $(2.4 \pm 0.8$ PPPY vs. $2.1 \pm 0.3$ PPPY; $p=0.038)$.

Similar to RA patients, having any access restrictions was not significantly associated with the odds of treatment effectiveness (OR 0.87, 95\% CI 0.67-1.14; $p=0.315$ ) or medication adherence (OR 0.82 , 95\% CI 0.64-1.06; $p=0.126$ ) among PsA patients receiving subcutaneous bDMARDs (Online Resource 3). However, the subset of patients whose access restrictions included step therapy had $27 \%$ lower odds of treatment effectiveness (OR 0.73, 95\% CI $0.54-0.98 ; p=0.037)$ compared with all other patients (PA only or no access restrictions), and $25 \%$ lower odds (OR $0.75,95 \%$ CI $0.56-1.02 ; p=0.06$ ) than patients who did not have access restrictions. The odds of medication adherence were 29\% lower (OR 0.71, 95\% 0.54-0.94; $p=0.017$ ) among PsA patients with step therapy requirements compared with all other patients, and $27 \%$ lower (OR 0.73, 95\% CI 0.55-0.96; $p=0.026$ ) when compared with patients with no restrictions.

Male sex and a higher number of rheumatologist visits in the baseline period were associated with higher odds of 
Table 4 Outpatient prescription drug use, healthcare resource utilization and costs for rheumatoid arthritis patients measured in the 12-month follow-up period

\begin{tabular}{|c|c|c|c|c|}
\hline & \multirow[t]{2}{*}{ All RA patients $[N=3993]$} & \multicolumn{2}{|l|}{ Access restrictions } & \multirow[t]{2}{*}{$p$ value (any vs. none) } \\
\hline & & Any $[N=1364]$ & None $[N=2629]$ & \\
\hline \multicolumn{5}{|l|}{ Medications } \\
\hline bDMARD & $3988(99.9)$ & $1361(99.8)$ & $2627(99.9)$ & 0.223 \\
\hline tsDMARD & $105(2.6)$ & $29(2.1)$ & $73(2.9)$ & 0.152 \\
\hline csDMARD & $2700(67.6)$ & $944(69.2)$ & $1756(66.8)$ & 0.122 \\
\hline Glucocorticoid & $2596(65.0)$ & $901(66.1)$ & $1695(64.5)$ & 0.320 \\
\hline Mean [SD] number of glucocorticoid claims & 4.2 [3.9] & $4.6[4.2]$ & $4.0[3.7]$ & $<0.001 * *$ \\
\hline Antidepressant & $1362(34.1)$ & $479(35.1)$ & $883(33.6)$ & 0.333 \\
\hline Mean $[\mathrm{SD}]$ number of antidepressant claims & $5.9[4.9]$ & $6.9[5.7]$ & $5.3[4.2]$ & $<0.001$ \\
\hline Antidiabetic & $440(11.0)$ & $176(12.9)$ & $264(10.0)$ & $0.006^{*}$ \\
\hline Antihyperlipidemic & $881(22.1)$ & $300(22.0)$ & $581(22.1)$ & 0.939 \\
\hline Antihypertensive & $1612(40.4)$ & $587(43.0)$ & $1025(39.0)$ & $0.013^{*}$ \\
\hline Pain medication & $2539(63.4)$ & 885 (64.9) & $1646(62.6)$ & 0.157 \\
\hline Opioid & $1817(45.5)$ & $627(46.0)$ & $1190(45.3)$ & 0.672 \\
\hline Mean [SD] number of opioid claims & $6.0[6.6]$ & $6.1[6.6]$ & $5.9[6.6]$ & 0.624 \\
\hline NSAID & $1655(41.1)$ & $579(42.4)$ & $1076(40.9)$ & 0.355 \\
\hline Mean [SD] number of NSAID claims & $3.8[3.2]$ & $4.2[3.6]$ & $3.5[2.9]$ & $<0.001 * *$ \\
\hline Other pain medication & $736(18.4)$ & $259(19.0)$ & $477(18.1)$ & 0.514 \\
\hline $\begin{array}{l}\text { Mean }[\mathrm{SD}] \text { number of other pain medication } \\
\text { claims }\end{array}$ & $3.6[3.5]$ & $3.7[3.5]$ & $3.5[3.5]$ & 0.327 \\
\hline \multicolumn{5}{|l|}{ Healthcare utilization } \\
\hline IP admission & $291(7.3)$ & $107(7.8)$ & $184(7.0)$ & 0.330 \\
\hline IP admission due to infection & $54(1.4)$ & $32(2.3)$ & $22(0.8)$ & $<0.001 * *$ \\
\hline Emergency room visits & $346(8.7)$ & $167(12.2)$ & $179(6.8)$ & $<0.001 * *$ \\
\hline RA-related office visits & $2534(63.5)$ & $842(61.7)$ & $1692(64.4)$ & 0.102 \\
\hline \multicolumn{5}{|l|}{ Healthcare costs PPPY } \\
\hline Medical costs (mean [SD]) & $\$ 4772[\$ 17,280]$ & $\$ 4488[\$ 16,834]$ & $\$ 4919[\$ 17,508]$ & 0.454 \\
\hline Medical costs (median) & $\$ 905$ & $\$ 802$ & $\$ 976$ & \\
\hline OP prescription costs (mean $[\mathrm{SD}]$ ) & $\$ 26,658[\$ 16,435]$ & $\$ 27,390[\$ 15,829]$ & $\$ 29,315[\$ 16,706]$ & $<0.001 * *$ \\
\hline OP prescription costs (median) & $\$ 28,596$ & $\$ 27,121$ & $\$ 29,380$ & \\
\hline Cost per OP prescription (mean [SD]) & $\$ 1191[\$ 1194]$ & $\$ 992[\$ 959]$ & $\$ 1294[\$ 1287]$ & $<0.001 * *$ \\
\hline Cost per OP prescription (median) & $\$ 872$ & $\$ 728$ & $\$ 955$ & \\
\hline Total healthcare costs (mean [SD]) & $\$ 33,429[\$ 24,025]$ & $\$ 31,878[\$ 23,420]$ & $\$ 34,235[\$ 24,298]$ & $0.003 *$ \\
\hline Total healthcare costs (median) & $\$ 32,063$ & $\$ 30,424$ & $\$ 32,638$ & \\
\hline
\end{tabular}

$D M A R D$ disease-modifying antirheumatic drug, $b D M A R D$ biologic DMARD, tsDMARD targeted synthetic DMARD, $c S D M A R D$ conventional synthetic DMARD, $S D$ standard deviation, NSAID nonsteroidal anti-inflammatory drug, $P P P Y$ per patient per year, $R A$ rheumatoid arthritis, $I P$ inpatient, $O P$ outpatient, $* p<0.05, * * p<0.001$

treatment effectiveness and medication adherence. While rural residency and baseline prescriptions for bDMARDs or tsDMARDs were associated with higher odds of medication adherence, a baseline diagnosis of anemia or a chronic respiratory condition was associated with lower odds of treatment effectiveness.

\section{Discussion}

In this retrospective claims-based cohort study, RA patients whose insurance plans required a stepped approach to the prescription of bDMARDs or tsDMARDs for the treatment of their disease had 17-19\% lower odds of treatment effectiveness compared with patients who did not have access restrictions or whose access restrictions only included PA. Examination of the components of treatment effectiveness revealed that differences in effectiveness between groups 
were driven by differences in medication adherence as those with step therapy requirements had 18-19\% lower odds of adherence compared with PA only or no restrictions. Among PsA patients, the decrease in the odds of treatment effectiveness associated with step therapy requirements was $25-27 \%$, driven by $27-29 \%$ lower odds of medication adherence for PsA patients with step therapy. These associations were weakened and lost statistical significance when patients with PA and patients with step therapy were combined into a single cohort, indicating that step therapy is a stronger deterrent to treatment effectiveness than PA alone.

Formulary restrictions may also be leading to higher utilization of healthcare resources. In this study, ER visits and inpatient admissions due to infections were higher among RA patients with access restrictions than those patients without access restrictions. Additionally, patients with access restrictions filled significantly more prescriptions for glucocorticoids and NSAIDs than patients without access restrictions, which could be an indication of poorly managed disease. However, total healthcare costs were significantly higher among RA patients without access restrictions, mainly due to higher prescription costs.

Among RA patients, poor adherence was the primary reason patients failed to meet the criteria for treatment effectiveness. In this study population, only $28.1 \%$ of patients met the criteria for adherence. Previous studies among RA patients treated with subcutaneous bDMARDs have reported adherence and effectiveness ranging from 16 to $81 \%$ depending on methodology, pharmaceutical agent, and patient population [26-28]. Other leading reasons for failing to meet the effectiveness criteria were the addition of a new csDMARD or switching to a new bDMARD or tsDMARD, which is consistent with a previous study using the Curtis algorithm to assess treatment effectiveness of adalimumab, etanercept, and infliximab among Medicaid patients [26].

A number of systematic reviews have assessed the current literature on medication adherence and treatment effectiveness of bDMARDS among patients with chronic immunemediated arthritis [29-31], with male sex and concomitant methotrexate use being the most consistent positive predictors of adherence and effectiveness among both RA and PsA patients. Although the current study did not examine concomitant use of other medications, the significant sex differences in both adherence and effectiveness observed here are consistent with prior studies. Previous use of a tumor necrosis factor inhibitor (TNFi) is a negative predictor of effectiveness with an alternate TNFi, but there is currently a lack of evidence on the impact of previous TNFi use on the subsequent effectiveness of bDMARDs with a different mechanism of action [30]. The impact of disease duration on response to treatment has been mixed [32-34], but is likely confounded by the negative impact of TNFi cycling on both adherence and effectiveness [35, 36]. Neither prior use of a TNFi specifically nor disease duration were examined in relation to bDMARD treatment patterns in this study. Future studies may be warranted that consider the effect of prior lines of therapy on the relationship between access restrictions and biologic treatment outcomes.

\subsection{Limitations}

There are several limitations to this study, many of which are common to all administrative claims-based studies. First, data collected for administrative purposes may not be collected and validated with the same rigor as data collected for research purposes; therefore, these datasets are subject to miscoding and undercoding, which may introduce bias or measurement error. Second, although we used multivariate analysis to control for differences in baseline characteristics, there are several known factors, such as clinical measures of disease severity at baseline, which influence treatment effectiveness that are not captured in administrative claims and therefore could not be controlled for. Although this analysis did assess the number of disease-related office visits as a proxy for disease severity, clinical data on disease severity were not available. Third, claims data only identify a dispensed medication and not whether the medication was administered or taken as prescribed, which may overestimate medication adherence. Lastly, this study used data from commercially insured patients, and the findings may not be generalizable to the uninsured or those with other insurance.

\section{Conclusions}

RA and PsA patients whose insurance plans restrict access to bDMARDs and/or tsDMARDs via step therapy have poorer treatment effectiveness during the 12 months following treatment initiation compared with patients in plans without access restrictions or whose plans only require PA; this difference is driven by lower drug adherence among patients with access restrictions. Future studies should investigate the impact of step therapy and other drug access restrictions on treatment effectiveness among other therapeutic areas and insurance types (e.g. Medicare).

Data Availability Statement The data that support the findings of this study are available from IBM Watson Health, but restrictions apply to the availability of these data, which were used under license for the current study and are therefore not publicly available. However, data are available from the authors upon reasonable request and with permission of IBM Watson Health. 
Acknowledgements Medical writing services were provided by Jessamine P. Winer-Jones, PhD, of IBM Watson Health. These services were paid for by Eli Lilly and Company.

Author contributions NB and BHJ contributed to the study design. All authors contributed to data collection, analysis and/or interpretation of data, and all authors approved the final manuscript.

\section{Compliance with Ethical Standards}

Conflict of interest Natalie Boytsov and Xiang Zhang are employed by Eli Lilly and Company. Barbara H. Johnson and Kristin A. Evans are employed by IBM Watson Health as consultants and received funding from Eli Lilly and Company to conduct this study.

Funding This study was funded by Eli Lilly and Company.

Ethics statement All study data were accessed with protocols compliant with US patient confidentiality requirements, including the Health Insurance Portability and Accountability Act (HIPAA) of 1996 regulations. As all databases used in the study are fully de-identified and compliant with the HIPPA, this study was exempt from Institutional Review Board approval.

Open Access This article is distributed under the terms of the Creative Commons Attribution-NonCommercial 4.0 International License (http://creativecommons.org/licenses/by-nc/4.0/), which permits any noncommercial use, distribution, and reproduction in any medium, provided you give appropriate credit to the original author(s) and the source, provide a link to the Creative Commons license, and indicate if changes were made.

\section{References}

1. Helmick CG, Felson DT, Lawrence RC, Gabriel S, Hirsch R, Kwoh CK, et al. Estimates of the prevalence of arthritis and other rheumatic conditions in the United States. Part I. Arthritis Rheum. 2008;58:15-25.

2. Myasoedova E, Crowson CS, Kremers HM, Therneau TM, Gabriel SE. Is the incidence of rheumatoid arthritis rising?: Results from olmsted county, minnesota, 1955-2007. Arthritis Rheum. 2010;62:1576-82.

3. Hunter TM, Boytsov NN, Zhang X, Schroeder K, Michaud K, Araujo AB. Prevalence of rheumatoid arthritis in the United States adult population in claim databases, 2004-2014. Rheumatol Int. 2017;37(9):1551-7.

4. Suresh E. Diagnosis of early rheumatoid arthritis: what the nonspecialist needs to know. J R Soc Med. 2004;97:421-4.

5. Birnbaum H, Pike C, Kaufman R, Marynchenko M, Kidolezi Y, Cifaldi M. Societal cost of rheumatoid arthritis patients in the us. Curr Med Res Opin. 2010;26:77-90.

6. Eberhardt K, Larsson BM, Nived K, Lindqvist E. Work disability in rheumatoid arthritis-development over 15 years and evaluation of predictive factors over time. J Rheumatol. 2007;34:481-7.

7. Prey S, Paul C, Bronsard V, Puzenat E, Gourraud PA, Aractingi $\mathrm{S}$, et al. Assessment of risk of psoriatic arthritis in patients with plaque psoriasis: a systematic review of the literature. J Eur Acad Dermatol Venereol. 2010;24:31-5.

8. Rachakonda TD, Schupp CW, Armstrong AW. Psoriasis prevalence among adults in the united states. J Am Acad Dermatol. 2014;70:512-6.

9. Husted JA, Gladman DD, Farewell VT, Cook RJ. Health-related quality of life of patients with psoriatic arthritis: a comparison with patients with rheumatoid arthritis. Arthritis Care Res. 2001;45:151-8.

10. Al Sawah S, Foster SA, Goldblum OM, Malatestinic WN, Zhu $\mathrm{B}$, Shi N, et al. Healthcare costs in psoriasis and psoriasis subgroups over time following psoriasis diagnosis. J Med Econ. 2017;20:982-90.

11. Kawalec P, Malinowski KP. The indirect costs of psoriatic arthritis: systematic review and meta-analysis. Expert Rev Pharmacoecon Outcomes Res. 2015;15:125-32.

12. Smolen JS, Landewé R, Bijlsma J, Burmester G, Chatzidionysiou $\mathrm{K}$, Dougados $\mathrm{M}$, et al. Eular recommendations for the management of rheumatoid arthritis with synthetic and biological diseasemodifying antirheumatic drugs: 2016 update. Ann Rheum Dis. 2017;76:960.

13. Upchurch KS, Kay J. Evolution of treatment for rheumatoid arthritis. Rheumatology. 2012;51:vi28-36.

14. Wolfe F, Hawley DJ, Cathey MA. Termination of slow acting antirheumatic therapy in rheumatoid arthritis: a 14-year prospective evaluation of 1017 consecutive starts. J Rheumatol. 1990;17:994-1002.

15. Pincus T, Marcum SB, Callahan LF. Longterm drug therapy for rheumatoid arthritis in seven rheumatology private practices: II. Second line drugs and prednisone. J Rheumatol. 1992;19:1885-94.

16. Singh JA, Saag KG, Bridges SL Jr, Akl EA, Bannuru RR, Sullivan MC, et al. 2015 American College of Rheumatology guideline for the treatment of rheumatoid arthritis. Arthritis Rheumatol. 2016;68:1-26.

17. Barton JL. Patient preferences and satisfaction in the treatment of rheumatoid arthritis with biologic therapy. Patient Prefer Adher. 2009;3:335-44.

18. Turner AP, Williams RM, Sloan AP, Haselkorn JK. Injection anxiety remains a long-term barrier to medication adherence in multiple sclerosis. Rehabil Psychol. 2009;54:116-21.

19. Curtis JR, Baddley JW, Yang S, Patkar N, Chen L, Delzell E, et al. Derivation and preliminary validation of an administrative claims-based algorithm for the effectiveness of medications for rheumatoid arthritis. Arthritis Res Ther. 2011;13:R155.

20. Curtis JR, Chastek B, Becker L, et al. Further evaluation of a claims-based algorithm to determine the effectiveness of biologics for rheumatoid arthritis using commercial claims data. Arthritis Res Ther. 2013;15:404.

21. Curtis JR, Schabert VF, Harrison DJ, et al. Estimating effectiveness and cost of biologics for rheumatoid arthritis: application of a validated algorithm to commercial insurance claims. Clin Ther. 2014;36:996-1004.

22. Curtis JR, Schabert VF, Yeaw J, et al. Use of a validated algorithm to estimate the annual cost of effective biologic treatment for rheumatoid arthritis. J Med Econ. 2014;17:555-66.

23. Curtis JR, Chastek B, Becker L, et al. Cost and effectiveness of biologics for rheumatoid arthritis in a commercially insured population. J Manag Care Spec Pharm. 2015;21:318-29.

24. Wu N, Bhurke S, Shah N, Harrison DJ. Application of a validated algorithm to estimate the effectiveness and cost of biologics for rheumatoid arthritis in the US pharmacy benefit manager context. Clinicoecon Outcomes Res. 2015;7:257-66.

25. Deyo RA, Cherkin DC, Ciol MA. Adapting a clinical comorbidity index for use with ICD-9-CM administrative databases. J Clin Epidemiol. 1992;45:613-9.

26. Oladapo A, Barner JC, Lawson KA, Novak S, Rascati KL, Richards KM, et al. Medication effectiveness with the use of tumor necrosis factor inhibitors among texas medicaid patients diagnosed with rheumatoid arthritis. J Manag Care Pharm. 2014;20:657-67.

27. Harley CR, Frytak JR, Tandon N. Treatment compliance and dosage administration among rheumatoid arthritis patients receiving 
infliximab, etanercept, or methotrexate. Am J Manag Care. 2003;9:S136-43.

28. Borah BJ, Huang X, Zarotsky V, Globe D. Trends in ra patients' adherence to subcutaneous anti-tnf therapies and costs. Curr Med Res Opin. 2009;25:1365-77.

29. Lopez-Gonzalez R, Leon L, Loza E, Redondo M, Garcia de Yebenes MJ, Carmona L. Adherence to biologic therapies and associated factors in rheumatoid arthritis, spondyloarthritis and psoriatic arthritis: a systematic literature review. Clin Exp Rheumatol. 2015;33:559-69.

30. Wijbrandts CA, Tak PP. Prediction of response to targeted treatment in rheumatoid arthritis. Mayo Clin Proc. 2017;92:1129-43.

31. Maneiro JR, Souto A, Salgado E, Mera A, Gomez-Reino JJ. Predictors of response to tnf antagonists in patients with ankylosing spondylitis and psoriatic arthritis: systematic review and metaanalysis. RMD Open. 2015;1(1):e000017.

32. Anderson JJ, Wells G, Verhoeven AC, Felson DT. Factors predicting response to treatment in rheumatoid arthritis: the importance of disease duration. Arthritis Rheum. 2000;43:22-9.
33. Gratacós J, Casado E, Real J, Torre-Alonso JC. Prediction of major clinical response (ACR35) to infliximab in psoriatic arthritis refractory to methotrexate. Ann Rheum Dis. 2007;66:493-7.

34. Iannone F, Fanizzi R, Scioscia C, Anelli MG, Lapadula G. Body mass does not affect the remission of psoriatic arthritis patients on anti-tnf- $\alpha$ therapy. Scand J Rheumatol. 2013;42:41-4.

35. Chastek B, Chen C-I, Proudfoot C, Shinde S, Kuznik A, Wei W. Treatment persistence and healthcare costs among patients with rheumatoid arthritis changing biologics in the USA. Adv Ther. 2017;34:2422-35.

36. Gottenberg J, Brocq O, Perdriger A, et al. Non-TNF-targeted biologic vs. a second anti-TNF drug to treat rheumatoid arthritis in patients with insufficient response to a first anti-TNF drug: a randomized clinical trial. JAMA. 2016;316:1172-80. 